\title{
Re-entrant hexagons in non-Boussinesq convection
}

\author{
By SANTIAGO MADRUGA ${ }^{1}$, HERMANN RIECKE \\ AND WERNER PESCH${ }^{2}$ \\ ${ }^{1}$ Department of Engineering Sciences and Applied Mathematics, Northwestern University, \\ Evanston, IL 60208, USA \\ ${ }^{2}$ Physikalisches Institut, Universität Bayreuth, D-95440 Bayreuth, Germany
}

(Received 14 October 2004 and in revised form 11 July 2005)

While non-Boussinesq hexagonal convection patterns are known to be stable close to threshold (i.e. for Rayleigh numbers $R \approx R_{c}$ ), it has often been assumed that they are always unstable to rolls for slightly higher Rayleigh numbers. Using the incompressible Navier-Stokes equations for parameters corresponding to water as the working fluid, we perform full numerical stability analyses of hexagons in the strongly nonlinear regime $\left(\epsilon \equiv\left(R-R_{c}\right) / R_{c}=O(1)\right)$. We find 're-entrant' behaviour of the hexagons, i.e. as $\epsilon$ is increased they can lose and regain stability. This can occur for values of $\epsilon$ as low as $\epsilon=0.2$. We identify two factors contributing to the re-entrance: (i) far above threshold there exists a hexagon attractor even in Boussinesq convection as has been shown recently and (ii) the non-Boussinesq effects increase with $\epsilon$. Using direct simulations for circular containers we show that the re-entrant hexagons can prevail even for sidewall conditions that favour convection in the form of competing stable rolls. For sufficiently strong non-Boussinesq effects hexagons even become stable over the whole $\epsilon$-range considered, $0 \leqslant \epsilon \leqslant 1.5$.

\section{Introduction}

Rayleigh-Bénard convection has served as an excellent paradigm for studying systems that spontaneously form spatial or spatio-temporal patterns. In recent years exciting results have been obtained for the stability and dynamics of structures that are connected with roll convection. We mention, in particular, spiral-defect chaos, which is obtained in convection of fluids with low Prandtl number, and domain chaos driven by the Küppers-Lortz instability in rotating systems. For a recent review see Bodenschatz, Pesch \& Ahlers (2000). In most of these investigations great care has been taken to keep the experimental systems close to the regime in which the Oberbeck-Boussinesq (OB) approximation is valid by minimizing the dependence of the fluid parameters on the temperature in order to avoid the appearance of cellular or hexagonal structures.

It has long been recognized that variations of the fluid parameters with the temperature, i.e. non-Oberbeck-Boussinesq (NOB) effects, break an up-down symmetry and therefore introduce otherwise prohibited mode interactions. The best-studied case is the resonant triad interaction among the three fundamental Fourier modes whose wavevectors form a hexagonal pattern. It renders the primary bifurcation to the hexagons transcritical with the consequence that the hexagons are preferred over rolls in the immediate vicinity of onset (Busse 1967). According to leading-order 
weakly nonlinear analysis, hexagons typically become unstable to rolls further above threshold where the amplitudes are larger and the resonant-triad interaction loses significance compared to interactions involving four modes (Palm 1960; Segel \& Stuart 1962; Segel 1965; Busse 1967; Palm, Ellingsen \& Gjevik 1967; Davis \& Segel 1968). This scenario of a transition from hexagons to rolls has been confirmed in a number of experimental investigations (Somerscales \& Dougherty 1970; Dubois, Bergé \& Wesfreid 1978; Richter 1978; Walden \& Ahlers 1981; Bodenschatz et al. 1991; Pampaloni et al. 1992). Very strong deviations from the Boussinesq approximation have not been investigated in as much detail. As the non-Boussinesq effects become stronger, the stability range of hexagons extends further into the strongly nonlinear regime. Moreover, for strong temperature-dependence of the viscosity square patterns can also become stable at large Rayleigh numbers (Busse 1989).

Recently, in two experiments a different scenario was identified. They showed that even if hexagons are unstable to rolls at intermediate values of the Rayleigh number, the hexagons can gain stability in the strongly nonlinear regime. In one experiment hexagons were observed at relatively high Rayleigh numbers $\left(\epsilon \equiv\left(R-R_{c}\right) / R_{c} \approx 3.5\right)$ under conditions in which the Oberbeck-Boussinesq approximation was quite well satisfied (Assenheimer \& Steinberg 1996). In that case hexagons with upflow and those with downflow in the centre are equivalent and both were observed to coexist in adjacent domains. A subsequent numerical stability analysis confirmed the existence of such stable OB-hexagons (Clever \& Busse 1996; Busse, Clever \& Grote 1999). They are characterized by a pronounced spatial concentration of the flow in the off-centre regions. To resolve the strong spatial variations of the hexagons in the plane, modes with relatively high wavenumbers, which are neglected in the familiar amplitude equations, need to be retained (Busse \& Clever 1999).

In the other experiment, which used $\mathrm{SF}_{6}$ near the thermodynamic critical point as the working fluid, it was found that the hexagons that arise in the immediate vicinity of the onset of convection can become unstable as the Rayleigh number is increased and then restabilize again at higher Rayleigh numbers, $\epsilon=O(1)$ (Roy \& Steinberg 2002). This restabilization was termed 're-entrance'. As the non-Boussinesq effects were increased the intermediate $\epsilon$-range over which rolls were the preferred planform shrank and eventually hexagons were found to dominate rolls from onset all the way to $\epsilon=O(1)$. Since the re-entrant hexagons have been observed even for moderate NOB-effects, the restabilization at larger $\epsilon$ has been attributed by Roy \& Steinberg (2002) to the high compressibility of $\mathrm{SF}_{6}$ near its critical point.

In previous numerical stability calculations of rotating non-Boussinesq convection in water it was found that hexagons can be linearly stable over the whole range $0 \leqslant \epsilon \leqslant 1$ (Young, Riecke \& Pesch 2003). In the strongly nonlinear regime $\epsilon=O(1)$ a chaotic state ('whirling chaos') was obtained in which individual hexagonal cells oscillate or rotate, often inducing the nucleation of additional cells. Even in the presence of lateral walls, which typically induce the nucleation of rolls, this hexagonbased spatio-temporally chaotic state was found to persist. In that investigation the mechanism that is responsible for the linear stability of the hexagons over a fairly wide range in $\epsilon$ was, however, not understood.

In the present paper we identify easily accessible parameter regimes of nonBoussinesq convection in which strongly nonlinear hexagons are linearly stable. We point out two mechanisms that contribute to their stability. First, the mechanism that stabilizes OB-hexagons at larger Rayleigh numbers also enhances the stability of the NOB-hexagons. Second, with increasing Rayleigh number the temperature difference across the fluid layer increases and with it the strength of the NOB-effects. A simple 
weakly nonlinear model shows that this effect alone can be sufficient to lead to a restabilization of the hexagons at relatively low values of $\epsilon$, which experimentally leads to re-entrant hexagons. Together, these two mechanism can lead to a restabilization for values of $\epsilon$ as low as $\epsilon=0.2$. Since our computations are based on water as a working fluid (cf. Young et al. 2003), which is essentially incompressible in the investigated regime, it is clear that high compressibility is not a necessary condition to obtain re-entrant hexagons.

The paper is organized as follows. In $\S 2$ we briefly review the basic equations that we use, pointing out in which way our computations focus on weakly nonBoussinesq, but strongly nonlinear convection. In $\S 3$ we present our results for the linear stability regimes of hexagonal and roll patterns. The two mechanisms leading to restabilization are discussed in $\S 4$. Direct simulations of the temporal evolution for boundary conditions mimicking circular containers are discussed in $\S 5$. Conclusions follow in $\S 6$.

\section{Basic equations}

We consider a horizontal fluid layer of thickness $d$, density $\rho$, kinematic viscosity $\nu$, heat conductivity $\lambda$, and specific heat $c_{p}$. The layer is infinite in the horizontal direction and is limited in the vertical direction by two horizontal rigid plates with high thermal conductivity. The system is heated from below (at temperature $T_{1}$ ) and cooled from above (at temperature $T_{2}<T_{1}$ ). The governing equations expressing the balance of momentum, mass, and energy are (Chandrasekhar 1961)

$$
\begin{aligned}
\partial_{t}\left(\rho u_{i}\right)+\partial_{j}\left(\rho u_{j} u_{i}\right) & =-\partial_{i} p-\rho g \delta_{i 3}+\partial_{j}\left(v \rho\left(\partial_{i} u_{j}+\partial_{j} u_{i}\right)\right), \\
\partial_{t} \rho+\partial_{j}\left(\rho u_{j}\right) & =0, \\
\partial_{t} T+u_{j} \partial_{j} T & =\frac{1}{\rho c_{p}} \partial_{j}\left(\lambda \partial_{j} T\right) .
\end{aligned}
$$

Here $\boldsymbol{u}=\left(u_{1}, u_{2}, u_{3}\right)$ is the fluid velocity, $T$ the temperature, $p$ the pressure, $g$ the acceleration due to gravity, and $\delta_{i j}$ is the Kronecker delta. Summation over repeated indices is implied. The origin of a Cartesian coordinate system with the $z$-axis perpendicular to the horizontal plates is fixed in the middle of the layer. As usual, viscous heating and volume viscosity effects can safely be neglected.

Realistic rigid boundary conditions are taken at the two boundaries for the velocity,

$$
\boldsymbol{u}=0 \text { at } z= \pm \frac{d}{2}
$$

and fixed values for the temperature

$$
\begin{aligned}
& T=T_{1} \equiv T_{0}+\frac{\Delta T}{2} \text { at } z=-\frac{d}{2}, \\
& T=T_{2} \equiv T_{0}-\frac{\Delta T}{2} \text { at } z=+\frac{d}{2} .
\end{aligned}
$$

Here $T_{0}=\left(T_{1}+T_{2}\right) / 2$ denotes the mean temperature and $\Delta T=\left(T_{1}-T_{2}\right)>0$ is the temperature difference across the layer. We assume an experimental procedure in which $T_{0}$ is kept constant while the main control parameter $\Delta T$ is varied.

We focus in this work on weakly non-Boussinesq convection, so we keep the temperature dependence of the various fluid properties to leading order, and expand 
them about the mean temperature $T_{0}$ in line with Busse's convention (Busse 1967),

$$
\begin{aligned}
\frac{\rho(T)}{\rho_{0}} & =1-\bar{\gamma}_{0} \frac{T-T_{0}}{T_{s}}\left(1+\bar{\gamma}_{1} \frac{T-T_{0}}{T_{s}}\right)+\cdots, \\
\frac{\nu(T)}{\nu_{0}} & =1+\bar{\gamma}_{2} \frac{T-T_{0}}{T_{s}}+\cdots, \\
\frac{\lambda(T)}{\lambda_{0}} & =1+\bar{\gamma}_{3} \frac{T-T_{0}}{T_{s}}+\cdots, \\
\frac{c_{p}(T)}{c_{p 0}} & =1+\bar{\gamma}_{4} \frac{T-T_{0}}{T_{s}}+\cdots,
\end{aligned}
$$

where $\rho_{0}, \nu_{0}, \lambda_{0}$, and $c_{p 0}$ denote the values of the respective quantities at the mean temperature $T_{0}$. The dots denote higher-order terms to be neglected. Introducing the thermal diffusivity $\kappa_{0}=\lambda_{0} / \rho_{0} c_{p}$, the scaling temperature $T_{s}=v_{0} \kappa_{0} /\left(\alpha_{0} g d^{3}\right)$ is used to define the non-dimensionalized slopes of the density, viscosity, heat conductivity, and heat capacity at $T_{0}$ in terms of the $\bar{\gamma}_{i}$. For instance, the usual heat expansion coefficient at $T=T_{0}$ is given by $\alpha_{0}=\bar{\gamma}_{0} / T_{s}$. Beyond the Boussinesq approximation the curvature of $\rho(T)$ at $T_{0}$, which is proportional to $\bar{\gamma}_{0} \bar{\gamma}_{1} / T_{s}^{2}$, also comes into play.

To make the governing equations and boundary conditions dimensionless, the following scales are selected: for the length $d$, for the time $d^{2} / \kappa_{0}$, for the pressure $\rho_{0} v_{0} \kappa_{0} / d^{2}$, and for the temperature $T_{s}$. This gives rise to two dimensionless quantities: the Prandtl number $\operatorname{Pr}=v_{0} / \kappa_{0}$, and the Rayleigh number $R=\Delta T / T_{s}=$ $\alpha_{0} \Delta T g d^{3} /\left(v_{0} \kappa_{0}\right)$. Thus, we use in the following the dimensionless temperatures $\hat{T}=T / T_{s}$ and $\hat{T}_{0}=T_{0} / T_{s}$, heat conductivity $\hat{\lambda}=\lambda / \lambda_{0}$, density $\hat{\rho}=\rho / \rho_{0}$, kinematic viscosity $\hat{v}=v / \nu_{0}$, and specific heat $\hat{c}_{p}=c_{p} / c_{p 0}$. Finally, we write the equations in terms of the dimensionless momenta $v_{i}=\rho u_{i} d / \rho_{0} \kappa_{0}$ instead of the velocities. In the following we omit the hats for simplicity.

Since the fluid velocities are small compared to the sound velocity we make the anelastic approximation (Gough 1969) and neglect the time derivative in the continuity equation (2.2). This simplifies the computation considerably since it reduces the number of evolution equations. Furthermore, $v_{i}$ becomes a solenoidal field, which can be represented in the standard poloidal-toroidal decomposition by two velocity potentials (Busse 1989) automatically enforcing the mass conservation.

The (dimensionless) conduction solution $(v=0)$ of (2.3) with (2.5), (2.6), (2.7), (2.9), (2.10) is given by

$$
T_{\text {cond }}=T_{0}+R\left(-z-\frac{\gamma_{3}}{2}\left(z^{2}-\frac{1}{4}\right)+O\left(\gamma_{3}^{2}\right)\right) .
$$

We rewrite the temperature $T$ in terms of the deviation $\Theta$ from the conductive profile neglecting its $O\left(\gamma_{3}^{2}\right)$-contribution,

$$
\Theta=T-T_{\text {cond }}=T-T_{0}-R\left(-z-\frac{\gamma_{3}}{2}\left(z^{2}-\frac{1}{4}\right)\right) .
$$

We then obtain as the final dimensionless equations

$$
\begin{aligned}
\frac{1}{\operatorname{Pr}}\left(\partial_{t} v_{i}+v_{j} \partial_{j}\left(\frac{v_{i}}{\rho}\right)\right)= & -\partial_{i} p+\delta_{i 3}\left(1+\gamma_{1}\left(-2 z+\frac{\Theta}{R}\right)\right) \Theta \\
& +\partial_{j}\left[v \rho\left(\partial_{i}\left(\frac{v_{j}}{\rho}\right)+\partial_{j}\left(\frac{v_{i}}{\rho}\right)\right)\right],
\end{aligned}
$$




$$
\begin{aligned}
\partial_{j} v_{j} & =0, \\
\partial_{t} \Theta+\frac{v_{j}}{\rho} \partial_{j} \Theta & =\frac{1}{\rho c_{p}} \partial_{j}\left(\lambda \partial_{j} \Theta\right)-\gamma_{3} \partial_{z} \Theta-R \frac{v_{z}}{\rho}\left(1+\gamma_{3} z\right) .
\end{aligned}
$$

The dimensionless boundary conditions are

$$
\boldsymbol{v}(x, y, z, t)=\Theta(x, y, z, t)=0 \text { at } z= \pm \frac{1}{2} .
$$

The non-dimensionalized fluid parameters (2.7)-(2.10) are now

$$
\begin{gathered}
\rho(\Theta)=1-\gamma_{0}\left(-z+\frac{\Theta}{R}\right), \\
\nu(\Theta)=1+\gamma_{2}\left(-z+\frac{\Theta}{R}\right), \\
\lambda(\Theta)=1+\gamma_{3}\left(-z+\frac{\Theta}{R}\right), \\
c_{p}(\Theta)=1+\gamma_{4}\left(-z+\frac{\Theta}{R}\right),
\end{gathered}
$$

where we have introduced $\gamma_{i} \equiv \bar{\gamma}_{i} R=\bar{\gamma}_{i} \Delta T / T_{s}$, which can be expressed as follows:

$$
\gamma_{i}(\Delta T)=\gamma_{i}^{c}\left(1+\frac{\Delta T-\Delta T_{c}}{\Delta T_{c}}\right)=\gamma_{i}^{c}\left(\frac{R}{R_{c}}\right) .
$$

Here $\gamma_{i}^{c}=\bar{\gamma}_{i} \Delta T_{c} / T_{s}$ denotes the non-Boussinesq coefficients evaluated at the onset of convection, $T=T_{c}$, as used in Busse (1967). In our case $\gamma_{i}^{c}=\bar{\gamma}_{i} \Delta T_{c} / T_{s}$ can be approximated well using the Boussinesq value for the critical temperature difference, $\Delta T_{c}=1708 T_{s}$. In principle, the non-Boussinesq effects induce small corrections through a shift in the threshold, which are easily numerically computed, if necessary. Note that in contrast to $\bar{\gamma}_{i}$, the $\gamma_{i}(\Delta T)$ are linear in the main control parameter $\Delta T$ and therefore have to be adjusted in the nonlinear regime.

We consider the non-Boussinesq effects to be weak and in all material properties keep only the leading-order temperature dependence beyond the Boussinesq approximation. Therefore the $\gamma_{1}$-term appears explicitly in (2.13), while in all other terms it would constitute only a quadratic correction just like the terms omitted in (2.7)(2.10). Correspondingly, we expand the denominators in (2.13), (2.15) that contain material properties to leading order in $\gamma_{i}$. In analogy to Busse (1967), we further omit non-Boussinesq terms that contain cubic nonlinearities in the amplitudes $v_{i}$ or $\Theta$, as they arise from the expansion of the advection terms $v_{j} \partial_{j}\left(v_{i} / \rho\right)$ and $\left(v_{j} / \rho\right) \partial_{j} \Theta$ when the temperature dependence of the density is taken into account. Since we will be considering Rayleigh numbers up to twice the critical value, which implies enhanced non-Boussinesq effects, these approximations may lead to quantitative differences compared to the fully non-Boussinesq system, even though the temperature dependence of the material properties themselves may be described quite well by a linear (or quadratic in the case of the density) approximation. Unfortunately we are not aware of rigorous solutions of (2.13), (2.14), (2.15) that would allow quantitative tests of our approximation scheme for finite $\gamma_{i}$.

In the weakly nonlinear regime, hexagon and roll patterns are described by the amplitudes $A_{i}$ of the three dominant Fourier modes associated with the wavevectors $\boldsymbol{q}_{1}=q(1,0), \boldsymbol{q}_{2}=q / 2(-1, \sqrt{3}), \boldsymbol{q}_{3}=q / 2(-1,-\sqrt{3})$. Roll solutions correspond to $A_{1} \neq$ $0, A_{2}=A_{3}=0$ and hexagons to $A_{1}=A_{2}=A_{3} \neq 0$, while for mixed hexagon solutions 


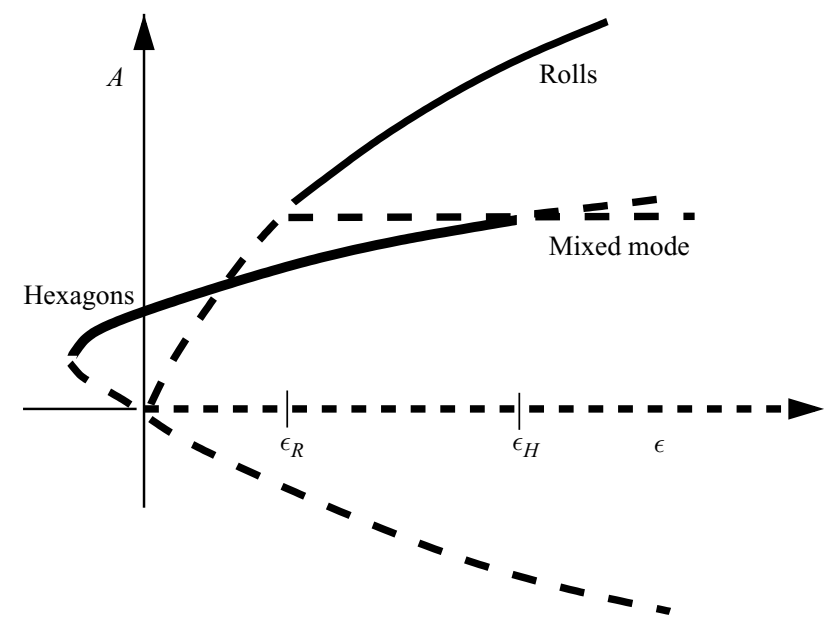

FIGURE 1. Sketch of the bifurcation diagram for hexagons and rolls in the weakly nonlinear regime of NOB-convection. The solid lines correspond to stable states and dashed lines to unstable ones. The unstable mixed mode destabilizes the hexagons and stabilizes the rolls.

$A_{1} \neq A_{2,3} \neq 0$. The amplitudes satisfy the well-known coupled amplitude equations (e.g. Cross \& Hohenberg 1993),

$$
\partial_{t} A_{1}=\epsilon A_{1}-\delta \bar{A}_{2} \bar{A}_{3}-g_{1}\left|A_{1}\right|^{2} A_{1}-g_{2}\left(\left|A_{2}\right|^{2}+\left|A_{3}\right|^{2}\right) A_{1}
$$

with the equations for $A_{2,3}$ obtained from (2.22) by cyclic permutation. The calculation of the coefficients $\delta, g_{i}$ involves vertical averages over certain products of the critical eigenvector components, which are obtained from a linearization of (2.13), (2.14), (2.15).

The quadratic coefficient $\delta$ arises from the NOB-effects. To leading order in $\gamma_{i}^{c}$ it was calculated first by Busse (1967) and is proportional to Busse's parameter $Q$, which is conventionally used as a measure for the strength of the NOB-effects. It is defined as

$$
Q=\sum_{i=0}^{4} \gamma_{i}^{c} \mathscr{P}_{i}
$$

where the $\mathscr{P}_{i}$ are certain linear functions of $\mathrm{Pr}^{-1}$, which can be found in the review article by Bodenschatz et al. (2000)†. Busse's parameter $Q$ characterizes the breaking of the up-down symmetry, which renders at most one of the two possible types of hexagons stable. Gases have a positive value of $Q$ and exhibit hexagons with downflow in the centre ( $g$-hexagons), whereas liquids have negative $Q$ and show hexagons with upflow ( $l$-hexagons). In the weakly nonlinear approach the cubic coefficients $g_{i}$ in (2.22) are evaluated with $\gamma_{i}^{c}=0$, which is consistent with the assumption of small $\gamma_{i}^{c}, \gamma_{i}^{c}=O\left(A_{i}\right)$. In the case of finite $\gamma_{i}^{c}$ the calculation can be refined along the lines presented by Plaut \& Pesch (1999).

The stability of weakly nonlinear roll and hexagon patterns is determined by a linear stability analysis of the various solutions of (2.22). The resulting bifurcation diagram is sketched in figure 1 for the case $g_{2}>g_{1}$ (cf. Busse 1967). The hexagons arise unstably from the conductive state in a transcritical bifurcation and become stable through a saddle-node bifurcation. When the heating, i.e. $\epsilon$, is increased they

$\dagger$ The expressions given on p. 742 of Bodenschatz et al. (2000) use the symbol $\mathscr{P}$ instead of $Q$ and correct a small error in Busse's calculation of $\mathscr{P}_{3}$. 
become unstable in a transcritical bifurcation at $\epsilon_{H}$ involving a mixed-mode solution. The rolls in turn are unstable at threshold and become stable through a pitchfork bifurcation at $\epsilon_{R}$, when the control parameter is increased.

We focus in this paper on the stability properties of the patterns in the strongly nonlinear regime. They are determined by a Galerkin expansion of all fields in (2.13), (2.14), (2.15) (see, for instance, Busse \& Clever 1979; Busse 1989). Their dependence on the vertical coordinate $z$ is captured by expanding them in appropriate combinations of trigonometric and Chandrasekhar functions in $z$ that satisfy the top and bottom boundary conditions (Chandrasekhar 1961; Busse 1967). In most of the computations we used $n_{z}=6$ modes for each field. With respect to the horizontal coordinates in the lateral directions we use a Fourier expansion on a hexagonal lattice. The Fourier wave vectors $\boldsymbol{q}$ are constructed as linear combinations of the hexagonal basis vectors $\boldsymbol{b}_{1}=q(1,0)$ and $\boldsymbol{b}_{2}=q(1 / 2, \sqrt{3} / 2)$ as $\boldsymbol{q}=m \boldsymbol{b}_{1}+n \boldsymbol{b}_{2}$ with the integers $m$ and $n$ in the range $\left|m \boldsymbol{b}_{1}+n \boldsymbol{b}_{2}\right| \leqslant n_{q} q$. The largest wavenumber is then $n_{q} q$ and the number of Fourier modes retained is given by $1+6 \sum_{j=1}^{n_{q}} j$. Typically we used $n_{q}=3$. Thus, solving the PDEs (2.13), (2.14), (2.15) is reduced to solving a system of nonlinear ODEs in time for the Galerkin expansion coefficients. The standard linear analysis of the ODEs yields the critical Rayleigh number $R_{c}$ as well as the critical wavenumber $q_{c}$. Both depend on the NOB-coefficients $\gamma_{i}^{c}$ which in turn depend on $R_{c}$. Thus, in principle one obtains an implicit equation for $\gamma_{i}^{c}$. The shift in the critical Rayleigh number away from the classical value $R_{c}=1708$ due to the NOB-effects is, however, quite small (less than $1 \%$ ) and therefore the resulting change in $\gamma_{i}^{c}$ is negligible. In this paper we therefore choose $\gamma_{i}^{c}$ corresponding to $R_{c}=1708$.

To investigate the nonlinear hexagon solutions, we start with the standard weakly nonlinear analysis to determine the coefficients of the coupled amplitude equations (2.22). To obtain the fully nonlinear solutions we need to solve the ODEs for the coefficients of the Galerkin expansion, which become a system of nonlinear algebraic equations in our stationary case. This is achieved with a Newton solver for which the weakly nonlinear solutions serve as convenient starting solutions. The solutions are tested for amplitude stability by monitoring the growth rates of linear perturbations of the expansion coefficients. Since the system is spatially periodic (characterized by Fourier modes with wavevectors $\boldsymbol{q}$ ) the possibility of side-band instabilities with respect to modes with wavevectors $\boldsymbol{q} \pm \boldsymbol{s}$ has to be considered as well. This is achieved by introducing Floquet multipliers $\exp (i s \cdot(x, y))$ in the Fourier ansatz for the linear perturbations of the Galerkin solutions.

We also study the dynamics of complex patterns that arise from instabilities of the periodic states. For that purpose we have extended our previously developed spectral code for the OB-equations (Pesch 1996; Bodenschatz et al. 2000) to include the NOBeffects in (2.13), (2.14), (2.15). It employs the same vertical modes as the Galerkin stability code but places the wavevectors of the Fourier modes on a rectangular rather than a hexagonal grid. To solve for the time dependence we have chosen a fully implicit scheme for the linear terms, whereas the nonlinear parts are treated explicitly (secondorder Adams-Bashforth method). The time step is typically taken to be $t_{v} / 500$, where $t_{v}$ is the vertical diffusion time. We have tested that the stability regimes obtained from the Galerkin analysis are consistent with the direct numerical simulations.

\section{Linear stability of hexagons}

Instead of extensive parameter studies, we present in this work specific interesting scenarios that should be experimentally realizable. We focus our investigation on 


\begin{tabular}{ccccccccc}
\hline$T_{0}\left[{ }^{\circ} \mathrm{C}\right]$ & $\Delta T_{c}\left[{ }^{\circ} \mathrm{C}\right]$ & $P r$ & $\gamma_{0}^{c}$ & $\gamma_{1}^{c}$ & $\gamma_{2}^{c}$ & $\gamma_{3}^{c}$ & $\gamma_{4}^{c}$ & $Q$ \\
20 & 20.63 & 6.93 & 0.0042 & 0.5693 & -0.5186 & 0.0649 & -0.0049 & -4.612 \\
25 & 15.16 & 6.10 & 0.0038 & 0.2952 & -0.3370 & 0.0434 & -0.0022 & -2.489 \\
28 & 12.94 & 5.68 & 0.0036 & 0.2122 & -0.2725 & 0.0352 & -0.0013 & -1.837 \\
32 & 10.74 & 5.18 & 0.0034 & 0.1440 & -0.2126 & 0.0273 & -0.0005 & -1.292 \\
36 & 9.12 & 4.76 & 0.0032 & 0.1023 & -0.1709 & 0.0216 & 0.0001 & -0.954 \\
40 & 7.87 & 4.38 & 0.0030 & 0.0755 & -0.1405 & 0.0173 & 0.0004 & -0.731 \\
50 & 5.75 & 3.62 & 0.0026 & 0.0400 & -0.0926 & 0.0104 & 0.0007 & -0.428 \\
60 & 4.42 & 3.05 & 0.0023 & 0.0245 & -0.0654 & 0.0064 & 0.0006 & -0.287
\end{tabular}

TABle 1. Values of the Prandtl number $\operatorname{Pr}$, non-Boussinesq coefficients $\gamma_{i}^{c}$, and Busse's parameter $Q$ for water at the onset of convection as a function of the mean temperature and the temperature difference. The liquid layer has a depth of $d=1.8 \mathrm{~mm}$.

water, which has a moderate Prandtl number and for which re-entrant hexagons should be readily accessible in convection cells with conventional layer thickness $d$ in a range of temperatures close to room temperature.

\subsection{Amplitude instabilities}

In our analysis, we first concentrate on spatially periodic solutions with the wavenumber fixed at the critical wavenumber and discuss their domains of existence and stability as a function of the control parameter $\epsilon=\left(R-R_{c}\left(\gamma_{i}^{c}\right)\right) / R_{c}\left(\gamma_{i}^{c}\right)$. We have chosen three different cells with thickness $d=1.5,1.8$, and $2.1 \mathrm{~mm}$, respectively. The case $d=1.8 \mathrm{~mm}$ is of particular interest since it has been studied in previous experiments (Pampaloni et al. 1992).

Table 1 gives the non-Boussinesq coefficients and the value of the non-Boussinesq parameter $Q$ at the onset of convection for a representative range of mean temperatures $T_{0}$ in a fluid layer of thickness $d=1.8 \mathrm{~mm}$. $\dagger$ As indicated before, $\gamma_{i}$ is linear in the temperature difference $\Delta T$ (see (2.21)) and therefore depends on $\epsilon$,

$$
\gamma_{i}=\gamma_{i}^{c}\left(1+\frac{R-R_{c}}{R_{c}}\right)=\gamma_{i}^{c}(1+\epsilon) .
$$

Note that with increasing mean temperature the critical temperature difference $\Delta T_{c}$ (given in the second column of table 1) decreases. Therefore the variation of the fluid properties across the layer at the critical temperature and with it the coefficients $\gamma_{i}^{c}$ also decrease with increasing temperature. Since we keep the mean temperature $T_{0}$ fixed when changing the Rayleigh number, the accessible range in $\epsilon$ is limited by the requirement that the temperature at the top plate, $T(z=d / 2)=T_{0}-\Delta T / 2$, be above freezing. It turns out that the full range $0 \leqslant \epsilon \leqslant 1$ is then only accessible for average temperatures $T_{0}$ above a certain temperature $T_{0}^{f}$, which is $20^{\circ} \mathrm{C}$ for $d=1.8 \mathrm{~mm}$.

Using the Galerkin method, we extend the weakly nonlinear result sketched in figure 1 (Busse 1967) to the strongly nonlinear regime. Figure 2 shows the resulting stability limits for hexagons and for rolls. As predicted by weakly nonlinear theory, the hexagons are linearly stable with respect to amplitude perturbations for very small $\epsilon$. For not too small values of the mean temperature $T_{0}$ and layer thickness $d$ they become unstable as the control parameter is increased. This instability corresponds to the transcritical bifurcation at $\epsilon_{H}$ in figure 1 . For the convection cells investigated 


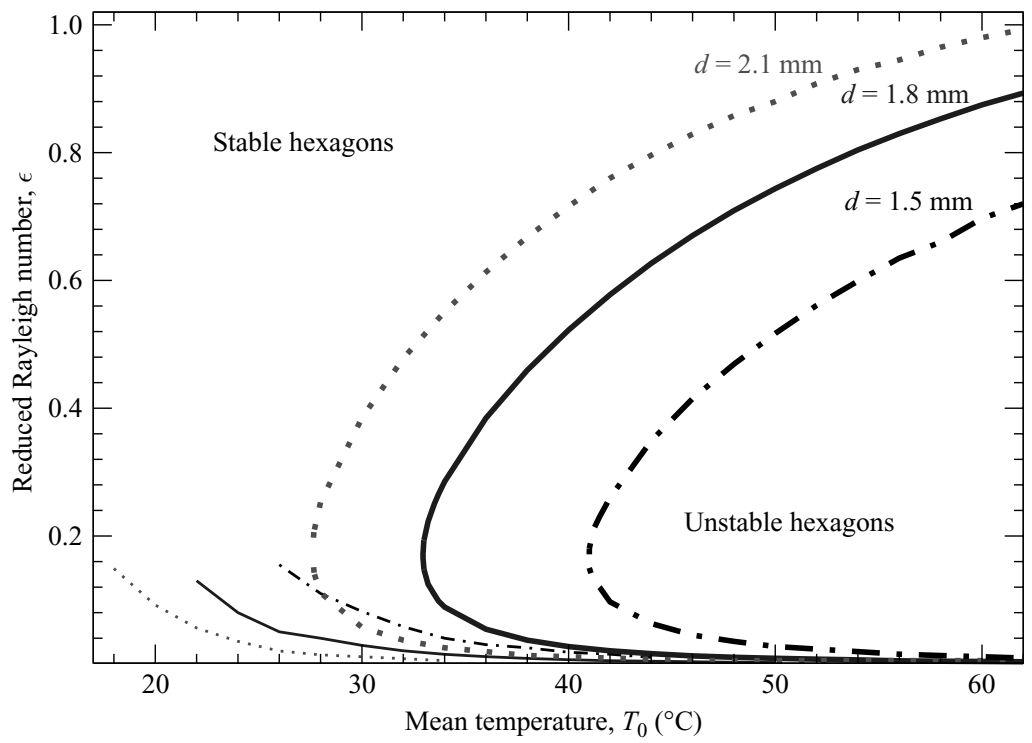

FIGURE 2. Stability regions for water with respect to amplitude perturbations for three fluid depths: $d=2.1 \mathrm{~mm}$ (dotted lines), $d=1.8 \mathrm{~mm}$ (solid lines), $d=1.5 \mathrm{~mm}$ (dot-dashed line). Thick curves: stability boundaries for hexagons. Thin curves: stability boundaries for rolls. For a given depth, rolls are stable above the thin line, and hexagons unstable in the inner region of the thick line. Stability limits are obtained for the critical wavenumber $q_{c}$.

here, the hexagon patterns then undergo a second steady bifurcation as the control parameter is increased further and become stable again. As the mean temperature or the layer thickness is decreased the critical heating and with it the non-Boussinesq effects increase. This shifts the point of restabilization to lower $\epsilon$ and the lower stability limit to higher $\epsilon$, decreasing the $\epsilon$-range over which the hexagons are unstable, until the two limits merge at a temperature $T_{m}$. For $T_{0}<T_{m}$ the hexagons are amplitude-stable over the whole range of $\epsilon$ considered $(0 \leqslant \epsilon \leqslant 1)$.

The restabilization of hexagons in the strongly nonlinear regime has been observed by Roy \& Steinberg (2002) in $\mathrm{SF}_{6}$ near the thermodynamical critical point, where it has been termed 're-entrance'. They argued that since the non-Boussinesq effects in that system are not very large near onset the re-entrance is due to the large compressibility of the fluid in this parameter regime. By assuming that the working fluid is incompressible, which is an excellent approximation for water, our computations show that high compressibility is not needed for re-entrance. The significance of the compressibility for the occurrence of the re-entrance has also been called into question recently by Ahlers (2005) (see his footnote 59) based on the work by Oh et al. (2004).

We have also computed the stability of rolls with respect to amplitude perturbations. The corresponding stability limits are indicated in figure 2 by thin lines. Note that it is not meaningful to extend these stability limits to lower values of $T_{0}$ than shown in figure 2 since then the temperature at the top plate would be below the freezing temperature of water. Below the thin lines rolls are unstable, but they become linearly stable when $\epsilon$ is increased beyond the lines. This stability limit corresponds to the pitchfork bifurcation at $\epsilon_{R}$ in figure 1. As the non-Boussinesq effects become stronger the stabilization of rolls is shifted to larger $\epsilon$. In contrast to the hexagons, for the convection cells investigated here the rolls do not undergo a second bifurcation, 

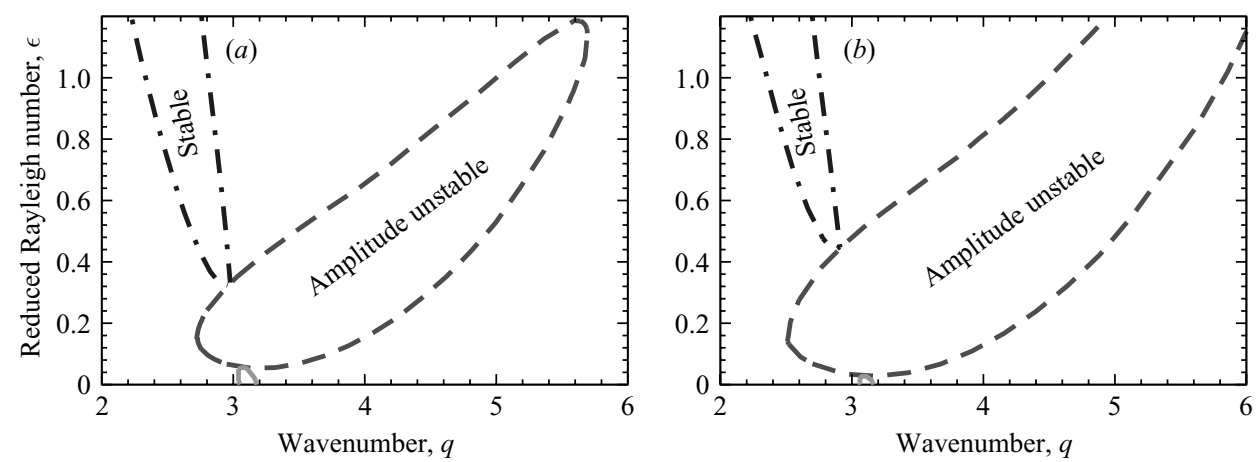

FIGURE 3. Stability regions for hexagons in water with respect to amplitude (dashed line) and side-band perturbations (dot-dashed line). The depth of the fluid layer is $d=1.8 \mathrm{~mm}$. (a) $T_{0}=36^{\circ} \mathrm{C},(b) T_{0}=40^{\circ} \mathrm{C}$. Hexagons are stable with respect to amplitude perturbations outside the dashed-line region, and stable with respect to side-band perturbations inside the dot-dashed line region.

which would destabilize them, and remain amplitude-stable up to the largest values of $\epsilon$ considered. For strong non-Boussinesq effects there is therefore a very large range of parameters over which the competing rolls and hexagons are both linearly amplitude-stable.

The amplitude-stability limits of the hexagons and rolls depend, of course, on their wavenumber. This is illustrated in figure 3 , where we fix the mean temperature $T_{0}$ and determine the stability limits of the hexagons as a function of their wavenumber $q$. Interestingly, for $T_{0}=36^{\circ} \mathrm{C}$ (figure $3 a$ ) the hexagons become more stable for small and for large wavenumbers and the instability region forms a bubble-like closed curve, outside which the hexagons are stable with respect to amplitude perturbations. These bubbles are hyper-surfaces in $q, \epsilon, T_{0}$-space. With decreasing non-Boussinesq effects (increasing $T_{0}$ ) the bubble grows and extends to larger values of $\epsilon$. Eventually, the upper part of the bubble is shifted to $\epsilon$-values beyond the range considered in this paper (figure $3(b)$ for $T_{0}=40^{\circ} \mathrm{C}$ ).

\subsection{Side-band instabilities}

Using the Galerkin method, we have studied the stability of the hexagons with respect to long- and short-wave perturbations as shown in figure 3. We find that over the whole range $0 \leqslant \epsilon \leqslant 1$ the only relevant side-band perturbations are long wave and steady, as is the case in the weakly nonlinear regime. The long-wave perturbations involve longitudinal and transverse phase modes, which can be described by two coupled evolution equations (Lauzeral, Metens \& Walgraef 1993; Hoyle 1995; Echebarria \& Pérez-García 1998). The stability limits obtained from the Galerkin analysis are shown in figure 3. In this parameter regime the stability region consists of two disconnected domains, reflecting the re-entrant nature of the hexagons. The stability domain near onset is very small and closes up as the amplitude stability limit is reached. This behaviour corresponds to that obtained from the weakly nonlinear theory (Lauzeral et al. 1993). In the re-entrant regime the stable domain opens up again in an analogous fashion when the amplitude-stability limit is passed. Note that the stability boundaries lean toward lower wavenumbers. Thus, stable re-entrant hexagonal patterns are expected to have wavenumbers below $q_{c}$.

As the mean temperature is lowered the bubble of the amplitude instability shrinks and eventually the bubble disappears (figure 4). The side-band stability limit then 


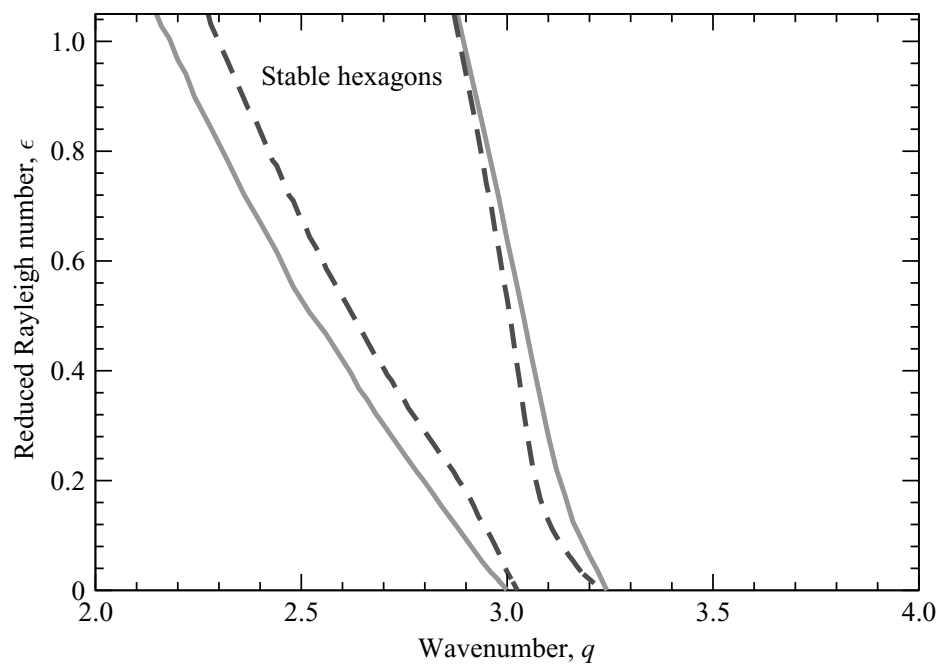

FIGURE 4. Stability regions for hexagons with respect to side-band pertubations, in water. The fluid depth is $d=1.8 \mathrm{~mm}$. Solid line: $T_{0}=28^{\circ} \mathrm{C}$. Dashed line: $T_{0}=32^{\circ} \mathrm{C}$.

reaches without interruption from the strongly nonlinear regime all the way down to threshold (more precisely to the saddle-node bifurcation of the hexagons). As the non-Boussinesq effects become yet stronger the range of stable wavenumbers widens.

\section{Origin of re-entrant hexagons}

At first the appearance of stable re-entrant non-Boussinesq hexagons seems quite surprising, in particular considering the relatively small values of $\epsilon$ for which the restabilization of the hexagons can occur. We have identified two major factors that contribute to their appearance. One is the fact that even in the Boussinesq case hexagons can be stable for sufficiently large Rayleigh numbers (above $\epsilon \approx 1$ ). They have been observed in convection experiments using $\mathrm{SF}_{6}$ close to its thermodynamical critical point as a working fluid where they nucleated in the cores of target and spiral patterns (Assenheimer \& Steinberg 1996). A subsequent numerical stability analysis confirmed the existence of stable OB-hexagons and attributed their appearance to the formation of plumes (Clever \& Busse 1996; Busse et al. 1999). The second factor contributing to the re-entrance of the non-Boussinesq hexagons is the increase of the non-Boussinesq effects with the Rayleigh number (cf. (3.1)).

Figure 5 provides a quantitative assessment of the importance of the two mechanisms contributing to the re-entrance of non-Boussinesq hexagons. Above the dasheddotted line hexagons with wavenumber $q_{c}$ become amplitude-stable in the Boussinesq case $\gamma_{i}=0$. This transition line corresponds to the stability limit of Boussinesq hexagons found earlier by Clever \& Busse (1996) (see their figure 4). The very slight dependence of the stability limit on the mean temperature is due to the variation of the Prandtl number with $T_{0}$, which decreases from $P r=5.4$ at $T_{0}=30{ }^{\circ} \mathrm{C}$ to $P=3.0$ at $T_{0}=60^{\circ} \mathrm{C}$. With the non-Boussinesq effects included, the stabilization of the hexagons occurs at lower values of the control parameter. The dashed line in figure 5 shows the resulting stability limits when the $\epsilon$-dependence of $\gamma_{i}$ is neglected, $\gamma_{i}=\gamma_{i}^{c}$, while the solid line denotes the stability limit with the dependence retained, $\gamma_{i}=\gamma_{i}^{c}(1+\epsilon)$. Thus, even when the non-Boussinesq effects are kept constant the stability limit connected 


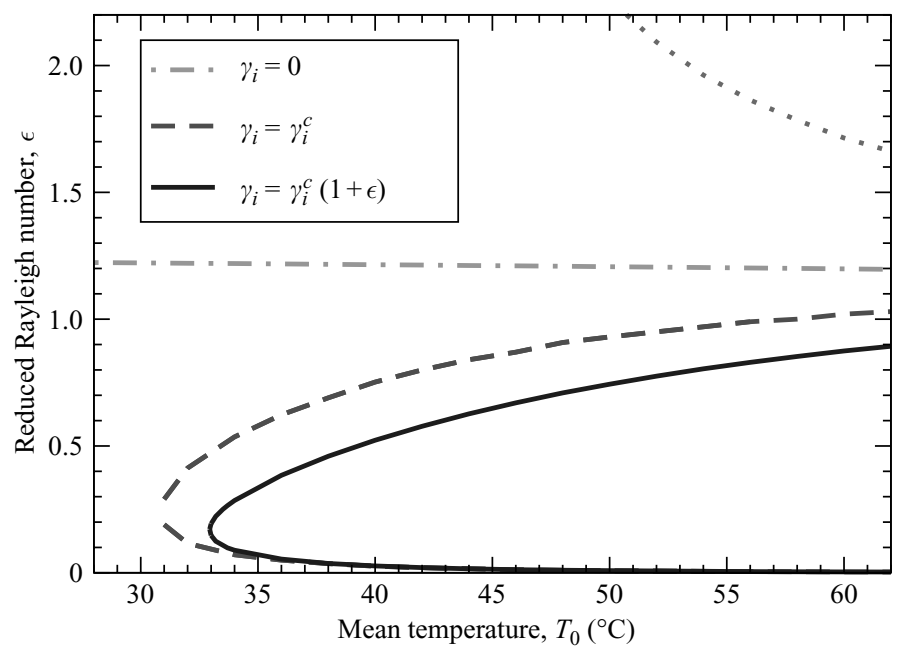

FIGURE 5. Stability regions for hexagons with respect to amplitude perturbations in water for $d=1.8 \mathrm{~mm}$. Solid line: stability limit when the dependence of $\gamma$ on the temperature is taken into account, $\gamma_{i}=\gamma_{i}^{c}(1+\epsilon)$. Dashed line: stability limit when the $\gamma_{i}$ are fixed to their critical value, $\gamma_{i}=\gamma_{i}^{c}$. OB-hexagons are stable above the dashed-dotted line, $\gamma_{i}=0$. Down-hexagons are stable above the dotted line for $\gamma_{i}=\gamma_{i}^{c}(1+\epsilon)$.

with the stabilization of Boussinesq hexagons merges with the usual low- $\epsilon$ stability limit when the non-Boussinesq effects become strong enough, i.e. at low temperatures.

Since in the Boussinesq case up- and down-hexagons are equivalent and become stable simultaneously it is to be expected that for weak non-Boussinesq effects both types of hexagons can become stable for large Rayleigh numbers, with the stabilization occurring, however, at different values of the Rayleigh number. For the up-hexagons, which in water are stable near onset, this stabilization corresponds to a re-entrance. The stabilization of the down-hexagons is indicated in figure 5 by a dotted line. As the non-Boussinesq effects become stronger the down-hexagons require ever higher Rayleigh numbers for stabilization.

Even though the $\epsilon$-dependence of the non-Boussinesq effects is not the central driving force for the re-entrance, it is instructive to discuss its effect on the hexagons briefly within a weakly nonlinear framework. We consider as a minimal model a slightly generalized version of (2.22) in which the quadratic coupling coefficient grows linearly with $\epsilon$,

$$
\partial_{t} A_{1}=\xi^{2}\left(\boldsymbol{n}_{1} \cdot \nabla\right)^{2} A_{1}+\epsilon A_{1}-(\delta+\mu \epsilon) \bar{A}_{2} \bar{A}_{3}-g_{1}\left|A_{1}\right|^{2} A_{1}-g_{2}\left(\left|A_{2}\right|^{2}+\left|A_{3}\right|^{2}\right) A_{1} .
$$

Note that such a linear correction of the quadratic resonance term has been considered previously in the context of hexagonal patterns in ferrofluids exposed to a magnetic field (Friedrichs \& Engel 2001). Compared to (2.22) we include a spatial gradient term involving the normal derivative $\boldsymbol{n}_{i} \cdot \nabla$ with $\boldsymbol{n}_{i}=\boldsymbol{q}_{i} /\left|\boldsymbol{q}_{i}\right|, i=1,2$, 3. It allows long-wave modulations of the amplitude that capture side-band instabilities.

To get a complete picture of the impact of the $\epsilon$-dependence of the non-Boussinesq effects within the framework of the simple model (4.1) we introduce a rescaled amplitude $\mathscr{A}=g_{1} A /(\delta+\mu \epsilon)$, a rescaled time $\hat{t}=(\delta+\mu \epsilon)^{2} t / g_{1}$, and a rescaled space variable $\hat{\boldsymbol{r}}=(\delta+\mu \epsilon) \boldsymbol{r} / \xi \sqrt{g_{1}}$. Equation (4.1) can then be written as

$$
\partial_{\hat{t}} \mathscr{A}_{1}=\left(\boldsymbol{n}_{1} \cdot \hat{\nabla}\right)^{2} \mathscr{A}_{1}+\hat{\epsilon} \mathscr{A}_{1}-\overline{\mathscr{A}}_{2} \overline{\mathscr{A}}_{3}-\left|\mathscr{A}_{1}\right|^{2} \mathscr{A}_{1}-\frac{g_{2}}{g_{1}}\left(\left|\mathscr{A}_{2}\right|^{2}+\left|\mathscr{A}_{3}\right|^{2}\right) \mathscr{A}_{1}
$$




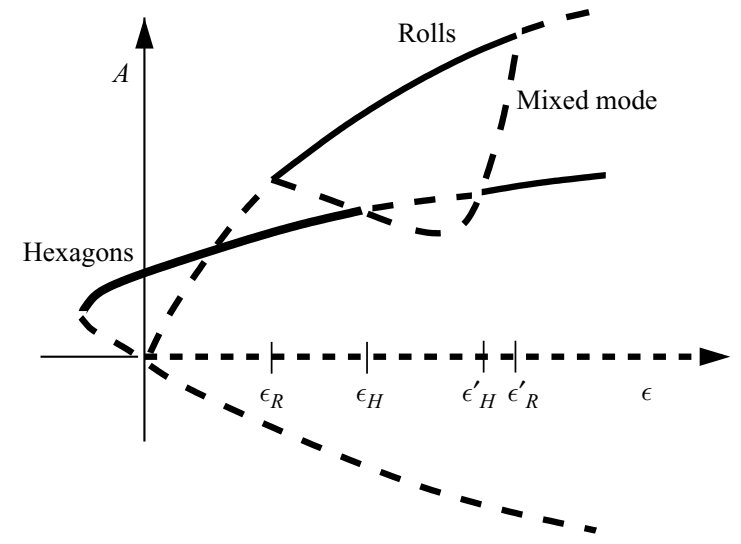

FIGURE 6. Sketch of the bifurcation diagram obtained from (4.1) (cf. figure 7 below for $k=0)$.

Here the rescaled control parameter is given by

$$
\hat{\epsilon}=\frac{\epsilon g_{1}}{(\delta+\mu \epsilon)^{2}},
$$

which implies that each value of $\hat{\epsilon}$ corresponds to two values of $\epsilon, \epsilon_{1,2}=\left(g_{1}-\right.$ $\left.2 \delta \mu \hat{\epsilon} \pm \sqrt{g_{1}^{2}-4 g_{1} \delta \mu \hat{\epsilon}}\right) / 2 \mu^{2} \hat{\epsilon}$.

For the case $g_{1}>0$ and $\mu \delta>0$, which is of interest here, a monotonic increase in $\epsilon$ is mapped into a non-monotonic change of $\hat{\epsilon}$ with the range $0 \leqslant \epsilon \leqslant \epsilon_{m} \equiv \delta / \mu$ mapped onto $0 \leqslant \hat{\epsilon} \leqslant g_{1} / 4 \delta \mu$ and the range $\epsilon_{m} \leqslant \epsilon<\infty$ mapped in a reverse fashion onto the same interval, $g_{1} / 4 \delta \mu \geqslant \hat{\epsilon}>0$. Thus, with increasing $\epsilon$ the standard bifurcation diagram sketched in figure 1 is traversed towards the right up to $\epsilon=\epsilon_{m}$. Note that the parameter $\epsilon$ in figure 1 plays the role of $\hat{\epsilon}$ in (4.2). As $\epsilon$ is increased further $\hat{\epsilon}$ decreases implying that the path through the bifurcation diagram in figure 1 is reversed. Thus, if $\epsilon_{m}$ is not too small the same mixed mode that is created in the bifurcation stabilizing the rolls and that destabilizes the hexagons at a larger value of $\epsilon$ in a transcritical bifurcation restabilizes the hexagons in a second transcritical bifurcation at $\epsilon=\epsilon_{H}^{\prime}$ and eventually disappears at the bifurcation that destabilizes the rolls again at $\epsilon=\epsilon_{R}^{\prime}$. This scenario is shown in the qualitative bifurcation diagram depicted in figure 6 . For larger values of $\mu$,

$$
\mu>\mu_{M} \equiv \frac{1}{2 \delta} \frac{\left(g_{1}-g_{2}\right)^{2}}{2\left(2 g_{1}+g_{2}\right)},
$$

$\hat{\epsilon}$ does not reach the stability limit of the hexagons and the hexagons remain linearly stable for all values of $\epsilon$. Specifically, for $\mu=\mu_{m}$ the two transcritical bifurcations at $\hat{\epsilon}_{H}$ and $\hat{\epsilon}_{H}^{\prime}$ coincide and the upper and lower stability limits in figure 5 merge, eliminating the amplitude-unstable regime of the hexagons.

Figure 7 gives the side-band stability limits obtained within the minimal model (4.1) for a typical case. The solid lines denote the transverse long-wave phase mode, while the dotted line marks the longitudinal phase mode. The bifurcation diagram shown in figure 6 corresponds to traversing this phase diagram at $q=q_{c}$. Note that the destabilization of rolls at $\epsilon_{R}^{\prime}$ is beyond the range of $\epsilon$ shown in figure 7. In principle, either of the phase modes can lead to an instability. Within (4.1), however, the longitudinal phase mode is relevant only for an extremely small range of $\epsilon$ near threshold. For small Prandtl numbers (4.1) would have to be extended to include a 


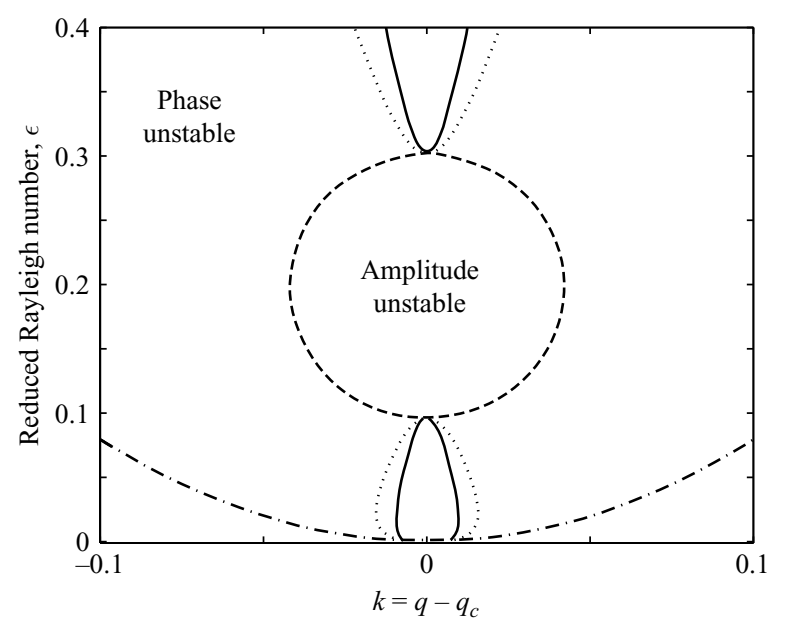

FIGURE 7. Amplitude and side-band stability limits based on the extended Ginzburg-Landauequation model (4.1) with $\xi=2.83, \delta=0.048, \mu=0.28, g_{1}=1$, and $g_{2}=1.45$. Shown are the neutral curve (dashed-dotted), the long-wave side-band stability limit (solid and dotted), and the amplitude stability limit (dashed). Hexagons are stable inside the solid lines.

mean flow. Then the longitudinal phase mode can dominate the transverse mode over significant portions of the stability limits (Young \& Riecke 2002; Semwogerere \& Schatz 2004).

Thus, the minimal model (4.1) captures qualitatively the restabilization of the hexagons, the merging of the two stability limits as the non-Boussinesq effects are increased, and the main features of the side-band instabilities obtained in the full stability computations described in $\S 3$. We have not found, however, the destabilization of rolls by a steady mode as it is suggested by the minimal model (cf. figure 6). Instead, we find at quite large $\epsilon$ an oscillatory instability.

The full numerical stability analysis presented in $\S 3$ displayed a tendency of the side-band-stable regions to shift to lower wavenumbers as $\epsilon$ is increased. In principle, this could be modelled phenomenologically by retaining nonlinear gradient terms in the minimal model (Bragard \& Velarde 1998; Echebarria \& Pérez-García 1998; Nuz, Nepomnyashchy \& Pismen 1998). We will not pursue this here. Instead we point out that in experiments and in numerical stability analyses the Boussinesq hexagons are found to be stable with respect to side-band instabilities only for wavenumbers noticeably below $q_{c}$ (Assenheimer \& Steinberg 1996; Clever \& Busse 1996). Considering the significance of the mechanism underlying the stability of the Boussinesq hexagons for the re-entrance of the non-Boussinesq hexagons, it is to be expected that the reduced wavenumber is a characteristic feature of this mechanism of re-entrance.

\section{Numerical simulations}

To make closer contact with the results that would be expected in experimental investigations we also performed direct numerical simulations of (2.13), (2.15). While our Galerkin approach uses realistic boundary conditions at the top and bottom plate it employs periodic boundary conditions in the lateral directions. Thus, these computations are able to predict instabilities that arise in the interior of the system, but they do not capture phenomena associated with the lateral walls. In most of the recent experiments circular containers have been used and typically it has been found 


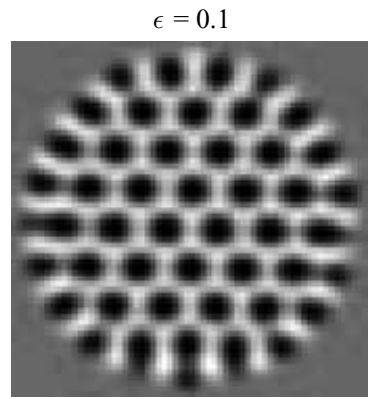

$\epsilon=1.0$

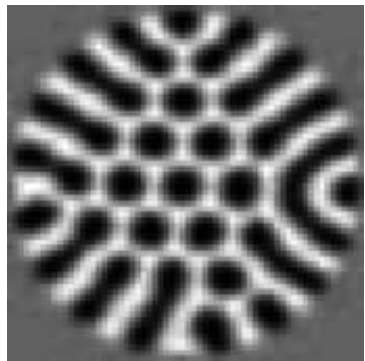

$\epsilon=0.2$

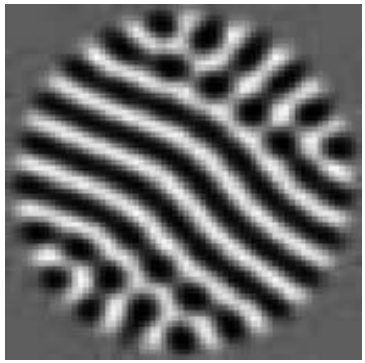

$\epsilon=1.2$

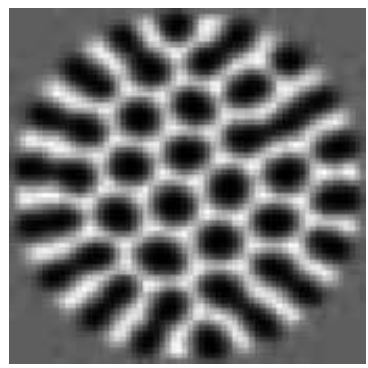

$\epsilon=0.6$

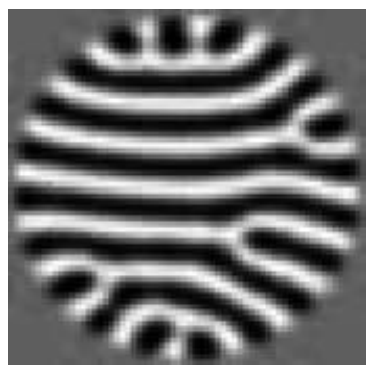

$\epsilon=1.4$

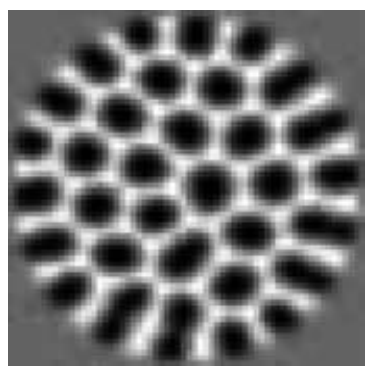

FIGURE 8. Succession of snapshots for $T_{0}=24^{\circ} \mathrm{C}$ in a circular cell of water of thickness $d=1.8 \mathrm{~mm}$. The diameter of the cell is $L=8 \times 2 \pi / q_{c}$ and the snapshots correspond to an integration time of $400 t_{v}$.

that with the walls rolls are preferred, which are predominantly either perpendicular or parallel to the wall depending on details of the sidewall conditions. Thus, even very close to onset, where in the non-Boussinesq case rolls are unstable to hexagons, in these experiments a narrow ring of roll-like structures arises.

A consequence of the wall-induced preference of rolls over hexagons is the experimental observation that the transition from hexagons to rolls does not occur at the Rayleigh number at which the hexagons become linearly unstable, but at lower Rayleigh numbers (Bodenschatz et al. 1991). The transition is therefore more appropriately interpreted as arising from a competition between rolls and hexagons, which are simultaneously linearly stable in that regime. The lowest-order GinzburgLandau equation (4.1) is variational and therefore, within this framework, the transition is expected to occur when the energy of the rolls becomes lower than that of the hexagons. The full Navier-Stokes equations are not variational and therefore further away from threshold it is more appropriate to discuss the transition in terms of the invasion of one state into the other with the velocity of the front separating the two states going through zero at the transition point.

In order to mimic the experimentally employed circular containers we apply a strong radial subcritical ramp in the Rayleigh number that suppresses any convection outside a certain radius. With such a boundary condition rolls perpendicular to this 'wall' are preferred (cf. Decker, Pesch \& Weber 1994, figure 1 in Bodenschatz et al. 2000). Snapshots of simulations in such a circular cell with diameter $L=8 \times 2 \pi / q_{c}$ and layer thickness $d=1.8 \mathrm{~mm}$ are shown in figure 8 . In all cases random initial conditions were used. Near threshold $(\epsilon=0.1)$ the cell is completely filled with regular hexagons, except for a narrow ring of roll-like structures that are driven by the boundaries. When the heating is increased $(\epsilon=0.2)$ rolls in the interior become linearly amplitude-stable 


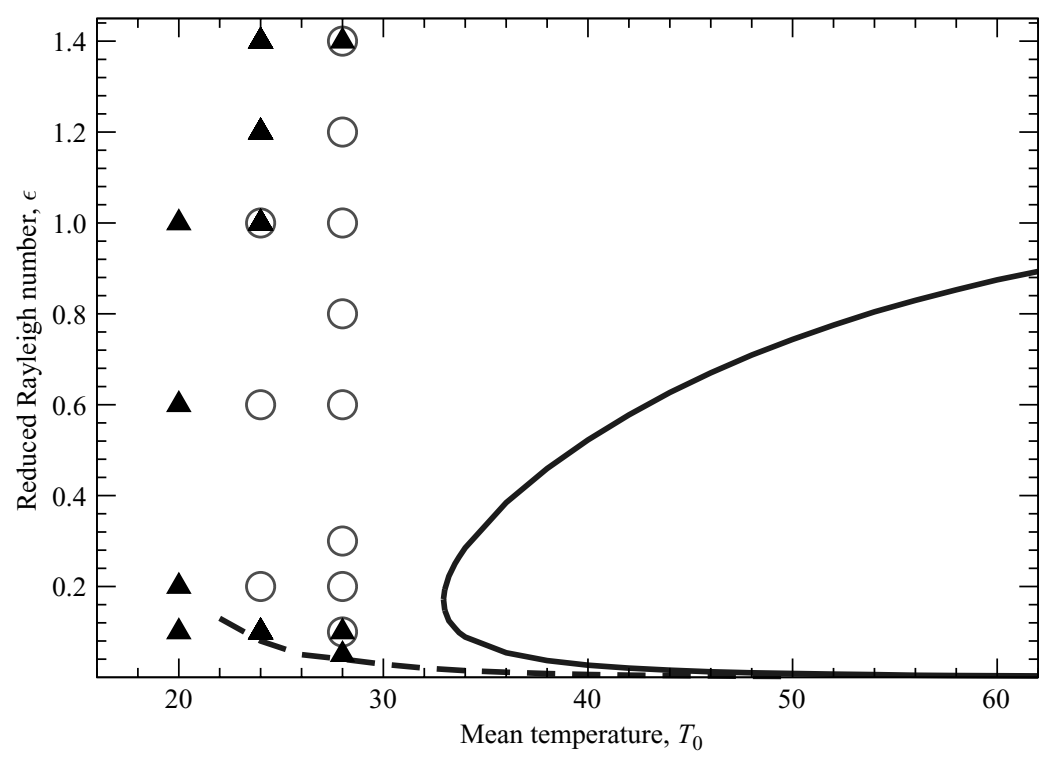

FIGURE 9. Results of simulations for a circular cell of thickness $d=1.8 \mathrm{~mm}$ and diameter $L=8 \times 2 \pi / q_{c}$. The simulations have been carried out for $T_{0}=20^{\circ} \mathrm{C}, 24^{\circ} \mathrm{C}, 28^{\circ} \mathrm{C}$. Circles correspond to rolls and triangles to hexagons. The stability limits for amplitude instabilities for hexagons (solid line) and for rolls (dashed line) are reproduced from figure 2.

(cf. figure 2) and the rolls that are driven by the boundary invade most of the cell. So far the scenario is very similar to the experimental observations shown in figure 4 of Pampaloni et al. (1992) for $T_{0}=28^{\circ} \mathrm{C}$ (see also figure 9). While the roll pattern persists near the boundaries for yet larger values of $\epsilon$, a domain of ordered hexagons appears in the centre of the cell for $\epsilon=O(1)$. The size of the inner domain of hexagons grows with increasing $\epsilon$ and for $\epsilon=1.4$ the hexagons essentially fill the whole convection cell. The growing of the hexagon domain with increasing $\epsilon$ can be understood to arise from a balance between the increasing tendency of the hexagon domains to invade domains of rolls on the one hand and the predominance of rolls near the boundaries on the other hand. It is worth noting that according to our computations Pampaloni et al. (1992) should have obtained stable re-entrant hexagons if they had gone to larger values of the Rayleigh number.

A comparison of the snapshot at $\epsilon=0.1$ with that at $\epsilon=1.4$ shows that the reentrant hexagons arising from random initial conditions have a smaller wavenumber than the hexagons that appear at threshold. This trend is consistent with the results of the side-band stability calculations (figures 3,4 ), which show that with increasing $\epsilon$ the wavenumber range of stable hexagons moves toward lower wavenumbers.

In figure 9 the results of a set of simulations in a circular cell with the same dimensions as in figure 8 are summarized for three mean temperatures, $T_{0}=20^{\circ} \mathrm{C}$, $T_{0}=24^{\circ} \mathrm{C}$, and $T_{0}=28^{\circ} \mathrm{C}$. Again random initial conditions are used. The triangles denote parameter values for which the final state consists mostly of regular hexagons, while the circles indicate a final roll state. When both patterns coexist over the course of the simulation $\left(t_{\max }=200 t_{v}\right)$ both symbols are plotted. The simulations show that for $T_{0}=20^{\circ} \mathrm{C}$ the NOB-effects are so strong that hexagons dominate rolls over the full range of $\epsilon$ studied. As seen before in figure 8 , for a mean temperature of $T_{0}=24^{\circ} \mathrm{C}$ an intermediate range of $\epsilon$ arises in which the final state consists of rolls. For $\epsilon=1.0$ 
rolls and hexagons coexist and for yet larger $\epsilon$ re-entrant hexagons appear. If the mean temperature is increased further to $T_{0}=28^{\circ} \mathrm{C}$ the same sequence: hexagons $\rightarrow$ rolls + hexagons $\rightarrow$ rolls $\rightarrow$ rolls + hexagons $\rightarrow$ hexagons is obtained. The only difference to the case $T_{0}=24^{\circ} \mathrm{C}$ is that the re-entrance is shifted to larger values of $\epsilon$.

To make contact with the linear stability analysis for periodic boundary conditions figure 9 also shows the stability limits for hexagons and rolls with solid and dashed lines, respectively. For the comparison with the results of the simulations in the circular container it is important to keep in mind that the point at which the hexagons and rolls have equal energy, or more generally, at which the velocity of fronts separating the two states changes sign, is not given by the linear stability limit of either the hexagons or the rolls, but rather lies somewhere between the two stability limits. It is therefore reasonable to expect that as the upper linear stability limit of hexagons is shifted to lower values of the Rayleigh number with decreasing mean temperature $T_{0}$, the Rayleigh number at which hexagons start to invade rolls also decreases and correspondingly the transition from rolls to hexagons in the presence of boundaries is also shifted to lower Rayleigh numbers.

\section{Conclusions}

In this paper we have studied non-Boussinesq convection in water for realistic parameters and boundary conditions. We have complemented numerical stability analyses for periodic boundary conditions with direct numerical simulations that mimic set-ups used in usual laboratory experiments. Our main result is the finding of re-entrant hexagons, i.e. we find that the hexagon patterns, which typically become unstable not far from threshold (Busse 1967), can regain stability further above threshold via a specific restabilization transition. For strong but realistic NOB-effects hexagons that become unstable at $\epsilon=0.15$ can become stable again at $\epsilon=0.2$. For yet stronger NOB-effects hexagons are amplitude-stable over the whole range of $\epsilon$ investigated $(\epsilon<1.5)$. This stabilization over the whole range of $\epsilon$ is not due to a shifting of the initial transition from hexagons to rolls to ever increasing $\epsilon$, but rather to the collision of this transition with the restabilization transition of the hexagons.

Re-entrant non-Boussinesq hexagons have been observed in convection experiments using $\mathrm{SF}_{6}$ near its thermodynamic critical point as a working fluid (Roy \& Steinberg 2002). Their restabilization has been attributed to the strong compressibility in this regime. Our computations, being based on water as the working fluid, demonstrate that compressibility is not necessary for this phenomenon. We show that in water the re-entrance is instead connected with the fact that even in the Boussinesq case hexagons can become stable for sufficiently large $\epsilon$ (Assenheimer \& Steinberg 1996; Clever \& Busse 1996) and with the increase of the non-Boussinesq effects with $\epsilon$. A simple amplitude-equation model capturing the latter aspect provides qualitative insight into the stability of the hexagons, including their side-band instabilities.

Reflecting the fact that Boussinesq hexagons are stable with respect to side-band perturbations only for low wavenumbers (Clever \& Busse 1996), we find that in water the wavenumber of the re-entrant hexagons is noticeably below $q_{c}$. In contrast, the strongly nonlinear hexagons found experimentally in $\mathrm{SF}_{6}$ do not exhibit this trend (Roy \& Steinberg 2002). Since we have found the same tendency towards lower wavenumbers also in gases away from the thermodynamic critical point (work in progress), one might speculate that the stabilization of the experimentally observed hexagons is due to a mechanism that differs from the mechanism of re-entrance discussed here. In the experimental $\mathrm{SF}_{6}$-system even small changes in the mean 
density can modify the non-Boussinesq effects significantly, which would strongly affect the transitions between rolls and hexagons (Ahlers 2005). Compared to $\mathrm{SF}_{6}$ near the critical point, water has the great advantage that the non-Boussinesq effects are much easier to control.

The connection between the re-entrant hexagons and the Boussinesq hexagons suggests that even in the non-Boussinesq case down-hexagons may become linearly amplitude-stable, albeit for yet higher Rayleigh numbers. We show that this is indeed the case. We have not investigated their side-band instabilities and it is not clear whether they can coexist with up-hexagons or whether domains of up-hexagons always invade domains of down-hexagons.

To address the stability and dynamics of the hexagon patterns in large-aspectratio systems with non-periodic boundary conditions we have also performed direct numerical simulations of the Navier-Stokes equations. By a suitable strong variation of the local Rayleigh number we have implemented a qualitatively convincing model of a circular container (Decker et al. 1994). For intermediate NOB-effects we confirm the experimentally observed scenario in the low- $\epsilon$ regime (Pampaloni et al. 1992). Our computations suggest that even in this experimental set-up re-entrant hexagons should have been accessible experimentally for somewhat stronger heating $(\epsilon \approx 1.5)$.

Since in experiments the sidewalls always induce roll convection the transition between rolls and hexagons occurs when the velocity of a front separating the two states changes sign and not at the stability limit of the hexagons. It is therefore not possible to study the amplitude-stability limit of the hexagons in such cells. We expect, however, that by forcing hexagonal patterns near the walls either by appropriate spacedependent heating (Semwogerere \& Schatz 2002) or by a suitably corrugated bottom plate (E. Bodenschatz, unpublished) hexagons can be stabilized near the walls and the bulk stability of hexagons with respect to rolls can be investigated.

The merging of the lower stability limit of hexagons with the restabilization line also provides an explanation for the large contiguous stability range that was found in rotating non-Boussinesq convection using water (Young et al. 2003). An interesting question is how the restabilization interacts with the Hopf bifurcation of the hexagons to oscillating hexagons, which is induced by rotation (Swift 1984; Soward 1985; Echebarria \& Riecke 2000; Madruga \& Pérez-García 2004).

We thank G. Ahlers for providing us with the code to determine the non-Boussinesq coefficient and for stimulating discussions. We also thank the computer center at the University of Bayreuth for providing us with unlimited access to its Siemens-Fujitsu vector computer and are, in particular, indebted to B. Winkler for years of assistance with running this machine and for providing storage for our huge amount of data. We gratefully acknowledge support by the office of Basic Energy Sciences at the Department of Energy (DE-FG02-92ER14303) and an NSF-SCREMS equipment grant (DMS-0322807).

\section{REFERENCES}

Ahlers, G. 2005 Experiments with Rayleigh-Bénard convection. In Dynamics of Spatio-temporal Cellular Structures - Henri Bénard Centenary Review (ed. I. Mutabazi, J. E. Wesfreid \& E. Guyon). Springer.

Assenheimer, M. \& Steinberg, V. 1996 Observation of coexisting upflow and downflow hexagons in Boussinesq Rayleigh-Bénard convection. Phys. Rev. Lett. 76, 756-759.

Bodenschatz, E., deBruyn, J. R., Ahlers, G. \& Cannell, D. 1991 Transitions between patterns in thermal convection. Phys. Rev. Lett. 67, 3078. 
Bodenschatz, E., Pesch, W. \& Ahlers, G. 2000 Recent developments in Rayleigh-Bénard convection. Annu. Rev. Fluid Mech. 32, 709-778.

Bragard, J. \& Velarde, M. G. 1998 Bénard-Marangoni convection: planforms and related theoretical predictions. J. Fluid Mech. 368, 165-194.

Busse, F. H. 1967 The stability of finite amplitude cellular convection and its relation to an extremum principle. J. Fluid Mech. 30, 625-649.

Busse, F. H. 1989 Fundamentals of thermal convection. In Mantle Convection: Plate Tectonics and Global Dynamics (ed. W. Peltier), p. 23. Gordon and Breach.

Busse, F. H. \& Clever, R. M. 1979 Instabilities of convection rolls in a fluid of moderate Prandtl number. J. Fluid Mech. 91, 319-335.

Busse, F. H. \& Clever, R. M. 1999 New patterns in Rayleigh-Bénard convection. In Des Phenomenes Critiques au Chaos (ed. P. Manneville), pp. 79-92. Saclay: DRECAM/SPEC CEA.

Busse, F. H., Clever, R. M. \& Grote, E. 1999 Weakly nonlinear and fully nonlinear pattern formation in isotropic convection layers. Chaos Solitons Fractals 10, 753-760.

Chandrasekhar, S. 1961 Hydrodynamic and Hydromagnetic Stability. Clarendon.

Clever, R. M. \& Busse, F. H. 1996 Hexagonal convection cells under conditions of vertical symmetry. Phys. Rev. E 53, R2037-R2040.

Cross, M. \& Hohenberg, P. 1993 Pattern formation outside of equilibrium. Rev. Mod. Phys. 65, $851-1112$.

Davis, S. H. \& Segel, L. A. 1968 Effects of surface curvature and property variation on cellular convection. Phys. Fluids 11, 470-476.

Decker, W., Pesch, W. \& Weber, A. 1994 Spiral defect chaos in Rayleigh-Bénard convection. Phys. Rev. Lett. 73, 648-651.

Dubois, M., Bergé, P. \& Wesfreid, J. 1978 Non-Boussinesq convective structure in water near 4-degrees-C. J. Physique 39, 1253-1257.

Echebarria, B. \& PÉrez-García, C. 1998 Phase instabilities in hexagonal patterns. Europhys. Lett. 43, 35-40.

EChebarRia, B. \& Riecke, H. 2000 Defect chaos of oscillating hexagons in rotating convection. Phys. Rev. Lett. 84, 4838-4841.

Friedrichs, R. \& Engel, A. 2001 Pattern and wave number selection in magnetic fluids. Phys. Rev. E 64, 021406.

Gough, D. O. 1969 The anelastic approximation in thermal convection. J. Atmos. Sci. 26, 448-456.

HoYLe, R. 1995 Nonlinear phase diffusion equations for the long-wave instabilities of hexagons. Appl. Math. Lett. 8(3), 81-85.

Lauzeral, J., Metens, S. \& Walgraef, D. 1993 On the phase dynamics of hexagonal patterns. Europhys. Lett. 24, 707-712.

Madruga, S. \& Pérez-García, C. 2004 Hexagonal patterns in a model for rotating convection. Int. J. Bifurcation Chaos 14, 107-117.

Nuz, A. E., Nepomnyashchy, A. A. \& Pismen, L. M. 1998 Stability of non-equilateral hexagonal patterns governed by generalized amplitude equations. Physica A 249, 179-183.

Oh, J., Ortiz de Zárate, J., Sengers, J. V. \& Ahlers, G. 2004 Dynamics of fluctuations in a fluid below the onset of Rayleigh-Bénard convection. Phys. Rev. E 69, 021106.

PALM, E. 1960 On the tendency towards hexagonal cells in steady convection. J. Fluid Mech. 8, 183-192.

Palm, E., Ellingsen, T. \& GJeviK, B. 1967 On occurrence of cellular motion in Bénard convection. J. Fluid Mech. 30, 651-661.

Pampaloni, E., Pérez-García, C., Albavetti, L. \& Ciliberto, S. 1992 Transition from hexagons to rolls in convection in fluids under non-Boussinesq conditions. J. Fluid Mech. 234, 393-416.

Pesch, W. 1996 Complex spatio-temporal convection patterns. Chaos 6, 348-357.

Plaut, E. \& Pesch, W. 1999 Extended weakly nonlinear theory of planar nematic convection. Phys. Rev. E 59, 1747-1769.

RichteR, F. M. 1978 Experiments on the stability of convection rolls in fluids whose viscosity depends on temperature. J. Fluid Mech. 89, 553-560.

Roy, A. \& Steinberg, V. 2002 ReEntrant hexagons in non-Boussinesq Rayleigh-Bénard convection: Effect of compressibility. Phys. Rev. Lett. 88, 244503. 
SEgEL, L. A. 1965 Non-linear interaction of finite number of disturbances to a layer of fluid heated from below. J. Fluid Mech. 21, 359-384.

Segel, L. A. \& Stuart, J. T. 1962 On the question of the preferred mode in cellular thermal convection. J. Fluid Mech. 13, 289-306.

Semwogerere, D. \& Schatz, M. F. 2002 Evolution of hexagonal patterns from controlled initial conditions in a Bénard convection experiment. Phys. Rev. Lett. 88, 054501.

Semwogerere, D. \& Schatz, M. F. 2004 Secondary instabilities of hexagonal patterns in a BénardMarangoni convection experiment. Phys. Rev. Lett. 93, 124502.

Somerscales, E. F. \& Dougherty, T. S. 1970 Observed flow patterns at initiation of convection in a horizontal liquid layer heated from below. J. Fluid Mech. 42, 755-768.

SowARD, A. M. 1985 Bifurcation and stability of finite amplitude convection in a rotating layer. Physica D 14, 227-241.

SwIFT, J. W. 1984 Convection in a rotating fluid layer. In Contemporary Mathematics, Vol. 28, p. 435. American Mathematical Society.

Walden, R. W. \& Ahlers, G. 1981 Non-Boussinesq and penetrative convection in a cylindrical cell. J. Fluid Mech. 109, 89-114.

Young, Y.-N. \& Riecke, H. 2002 Mean flow in hexagonal convection: stability and nonlinear dynamics. Physica D 163, 166-183.

Young, Y.-N., Riecke, H. \& Pesch, W. 2003 Whirling hexagons and defect chaos in hexagonal non-Boussinesq convection. New J. Phys. 5, 135. 